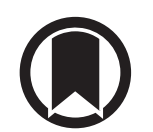

CrossMark

\title{
Severe Pulmonary Hypertension Management Across Europe (PHAROS): an ERS Clinical Research Collaboration
}

\author{
Olivier Sitbon (10 ${ }^{1,2,3}$, Abílio Reis $\mathbb{1}^{4}$, Marc Humbert (10) ${ }^{1,2,3}$, Anton Vonk \\ Noordegraaf ${ }^{5}$ and Marion Delcroix $\mathbb{B}^{6,7}$, on behalf of the PHAROS Clinical \\ Research Collaboration
}

Affiliations: ${ }^{1} \mathrm{APHP}, \mathrm{GH}$ Paris-Saclay, Hôpital Bicêtre, Service de Pneumologie, Centre de Référence de l'Hypertension Pulmonaire, Le Kremlin-Bicêtre, France. ${ }^{2}$ Université Paris-Saclay, Faculté de Médecine, Le Kremlin-Bicêtre, France. ${ }^{3}$ INSERM, UMR S999 "Pulmonary Hypertension: Pathophysiology and Innovative Therapies", Hôpital Marie-Lannelongue, Le Plessis-Robinson, France. "Pulmonary Vascular Disease Unit, Medicine Dept, Centro Hospitalar Universitário do Porto, Porto, Portugal. ${ }^{5}$ Dept of Pulmonary Medicine, Amsterdam UMC, Amsterdam, The Netherlands. ${ }^{6}$ Clinical Dept of Respiratory Diseases, Pulmonary Hypertension Center, UZ Leuven, Leuven, Belgium. ${ }^{7}$ BREATHE, Dept CHROMETA, KU Leuven, Leuven, Belgium. ${ }^{8}$ All institutions are members of ERN-Lung, PH core Network.

Correspondence: Olivier Sitbon, Service de Pneumologie, Hôpital Bicêtre, 78 rue du Général Leclerc, 94275 Le Kremlin-Bicêtre, France. E-mail: olivier.sitbonđu-psud.fr

@ERSpublications

The overall objective of the PHAROS CRC is to establish a platform for clinical research in pulmonary hypertension, in close collaboration with the ERN-Lung, able to coordinate future collaborative research in Europe https://bit.ly/2Rx2FTl

Cite this article as: Sitbon O, Reis A, Humbert M, et al. Severe Pulmonary Hypertension Management Across Europe (PHAROS): an ERS Clinical Research Collaboration. Eur Respir J 2020; 55: 2001047 [https:// doi.org/10.1183/13993003.01047-2020].

\section{Introduction}

The past 20 years have seen major advances in the understanding and treatment of pulmonary arterial hypertension (PAH; group 1 of the pulmonary hypertension $(\mathrm{PH})$ clinical classification) [1]. A strong basis of knowledge has been acquired in: 1) large randomised clinical trials for drug development; 2) national registries for epidemiology and outcome; and 3) smaller studies on the pathophysiological mechanisms of the disease. This knowledge has been reviewed at World Symposia on Pulmonary Hypertension (the most recent in 2018 [2]) and summarised in European Respiratory Society (ERS)/ European Society of Cardiology (ESC) clinical guidelines (the most recent in 2015 [3, 4]). We are, however, much less knowledgeable on specific aspects such as 1) the implementation of guidelines and access to therapies in different European countries; 2) the management of PH crises and progressive (acute on chronic) heart failure; and 3) other groups of $\mathrm{PH}$, such as $\mathrm{PH}$ due to lung diseases. Therapeutic strategies also need to be optimised, in particular regarding the combination of drugs, the use of anticoagulants, the place for new medications targeting different pathophysiological pathways, etc.

\section{Past achievements}

A few collaborative projects were initiated within the European Union (EU) Fifth and Sixth Framework Programmes. A first project on the "Early diagnosis and analysis of the genetic causes of primary pulmonary hypertension, a rare and life-threatening disease" started in 2002 (https://cordis.europa.eu/ 
project/rcn/64296_de.html), with the participation of seven European centres, and established a European registry and analysed further genetic causes of the disease. For early diagnosis of family members of index patients, they proposed non-invasive screening (Doppler echocardiography during exercise and/or hypoxia and ergo-spirometry) as well as genetic analysis [5]. A translational research collaboration started in 2006 with the EU Sixth Framework Programme funded multidisciplinary lung research project "Pulmotension, Pulmonary Hypertension: Functional Genomics and Therapy of Lung Vascular Remodelling", aimed to better understand and find a cure for PH. "Pulmotension" integrated 31 institutions at leading EU centres, in association with industrial partners in 12 European countries. This pan-European initiative allowed the collaborating researchers to investigate basic science questions in terms of clinical applicability and provided a unique potential for scientific breakthroughs, technological advances and new treatments for PH (final report https://cordis.europa.eu/result/rcn/47043_en.html). This collaboration has produced a number of high-impact publications. More recently, an International Consortium for Genetic studies in PAH (ICON PAH) has been established as a collaboration between the University of Cambridge, other European and US centres, and third party genome sequencing organisations, to discover the complete genetic architecture of PAH. To achieve this goal, the consortium members have established a platform for whole genome/exome sequencing and wish to share genetic data and analysis. Interestingly, the ERS recently funded a large-scale multicentre approach by using retrospective and prospective data of haemodynamics at rest and exercise to analyse the prognostic implications of exercise PH within a Clinical Research Collaboration (CRC) called the "pulmonary haemodynamics during exercise - research network" (PEX-NET) [6].

During the same period, individual countries have established national registries, recognising their essential role in bringing together the expertise that exists in individual centres. Major publications reporting on the epidemiology, outcome and prognostic factors in $\mathrm{PAH}$ have been produced from the French, Spanish, UK, Portuguese and German registries [7-12]. Validation of risk stratification tools has been a recent major advance for patient-tailored treatment of PAH [13-15]). Besides, a web-based dedicated software designed for the clinical management of PH patients (PAHTool ${ }^{\circ}$, Inovultus Ltd, Santa Maria da Feira, Portugal), has been developed and broadly implemented. It was created in 2008 and was the source of data for the first Portuguese PH registry involving five PH centres [12]. It has been used by the French Pulmonary Hypertension Network since September 2013, and now also in several PH centres all over the world. Since it is user friendly, it helps $\mathrm{PH}$ teams in their daily clinical activities (working according to the best practices, making clinical practice easier and helping in clinical decisions). In addition, it allows the construction of a database for information about clinical activities, results of clinical management, epidemiological investigation and research.

To further standardise the therapeutic approach to $\mathrm{PH}$ patients, educational programmes have been developed with scientific societies and academies, such as the annual "Pulmonary Hypertension and Pulmonary Vascular Disease course" of the ERS, the "Pulmonary Hypertension course" of the ESC, the University of Bologna "International master's degree in Pulmonary Vascular Diseases", and the World Symposia on Pulmonary Hypertension [2] that are held every 5 years.

In addition to its missions of drug development and clinical trials, the pharmaceutical industry has also engaged with investigators from the USA to develop patient reported outcome (PRO) tools, such as the Pulmonary Arterial Hypertension-Symptoms and Impact Questionnaire (PAH-SYMPACT) [16], developed according to the 2009 US Food and Drug Administration PRO guidance. In parallel, the Pulmonary Hypertension Association UK developed the emPHasis-10 quality-of-life questionnaire in collaboration with nationally designated specialist PH centres in the UK and Ireland [17].

\section{Current landscape}

Despite all these pioneering initiatives, we missed a coordinating structure in $\mathrm{PH}$, comprising members from European patient organisations, academic institutions and pharmaceutical companies, which would care for the collaborative project's prioritisation and sustainability. It would be set out to build on the existing set of individual PH-related activities across Europe to develop a comprehensive approach to $\mathrm{PH}$ research and to set development priorities, linking basic, translational and clinical science, health service innovation and drug development. European Reference Networks (ERNs), involving healthcare providers across Europe, and aiming to facilitate discussion on complex or rare diseases and conditions that require highly specialised treatment and concentrated knowledge and resources, were approved in December 2016 by the Board of EU Member States. Members are expected to develop and disseminate care guidelines, promote common treatment approaches, enhance cross-border access to diagnosis and treatment, initiate and support registries, and assemble sufficiently large cohorts for clinical studies, drug development and natural history studies. It is obvious that some of the ERN missions match ERS CRC aims, described in a recent editorial in the European Respiratory Journal [18]: 1) promoting the exchange of research ideas among clinicians and 
affiliated scientists in Europe and/or globally; 2) building an infrastructure for prospective clinical research; 3) securing additional funding through national and EU funding streams; and 4) facilitating the planning, implementation, evaluation and publication of clinical and translational studies at pan-European level and beyond. Synergies can be built, since ERNs' financial resources are currently directed towards the development of tools for care and patient data sharing, such as the Clinical Patient Management System.

With the creation of a dedicated Assembly for Pulmonary Vascular Diseases in 2018 (Assembly 13) [19] and the endorsement of the PHAROS (severe Pulmonary Hypertension mAnagement acROSs europe) $\mathrm{CRC}$, the ERS demonstrated a strong willingness to support education and research in PH. Along the same lines and in the context of the strong relationship established between ERN-Lung (ERN dedicated to rare lung diseases) and the ERS [20], PHAROS is devoted to the organisation and coordination of research projects involving the 17 healthcare providers (HCP) of the PH core of ERN-Lung (figure 1).

\section{Aims of PHAROS}

The overall objective of the PHAROS CRC is to establish a platform for clinical research in $\mathrm{PH}$, in close collaboration with the ERN-Lung, able to coordinate future collaborative research on topics not of immediate interest for the industry, such as pragmatic trials without immediate financial return, concerning care organisation or less prevalent $\mathrm{PH}$ subgroups, and to facilitate and accelerate the translation of basic research findings to the clinic. Ultimately, the purpose is also to build a clinical trial network providing expert opinion on feasibility and priority for industry-sponsored randomised clinical trials and to raise standards of research and harmonise standard operating procedures across the network of centres in Europe, working together with patients to prioritise their needs.

The specific aims of the 3-year PHAROS project are as follows: 1) to establish an inventory of existing national and local $\mathrm{PH}$ registries and look at interoperability to exchange data for clinical research projects; 2) to evaluate patient access to care across Europe by establishing an inventory of centre expertise (data collected by the centres in the application process for ERN-Lung), access to advanced therapies (parenteral prostanoids and lung transplantation), and use of PROs (PAH-SYMPACT or emPHasis-10), with special interest for care pathways and cross-border care; 3) to study progressive right heart failure in $\mathrm{PH}$; and 4) to study severe $\mathrm{PH}$ in patients with lung diseases.

A survey on local patient data registries of the $17 \mathrm{HCP}$ involved in the ERN-Lung PH core revealed important disparities between centres (figure 2). If the majority of the registries recorded all $\mathrm{PH}$ groups

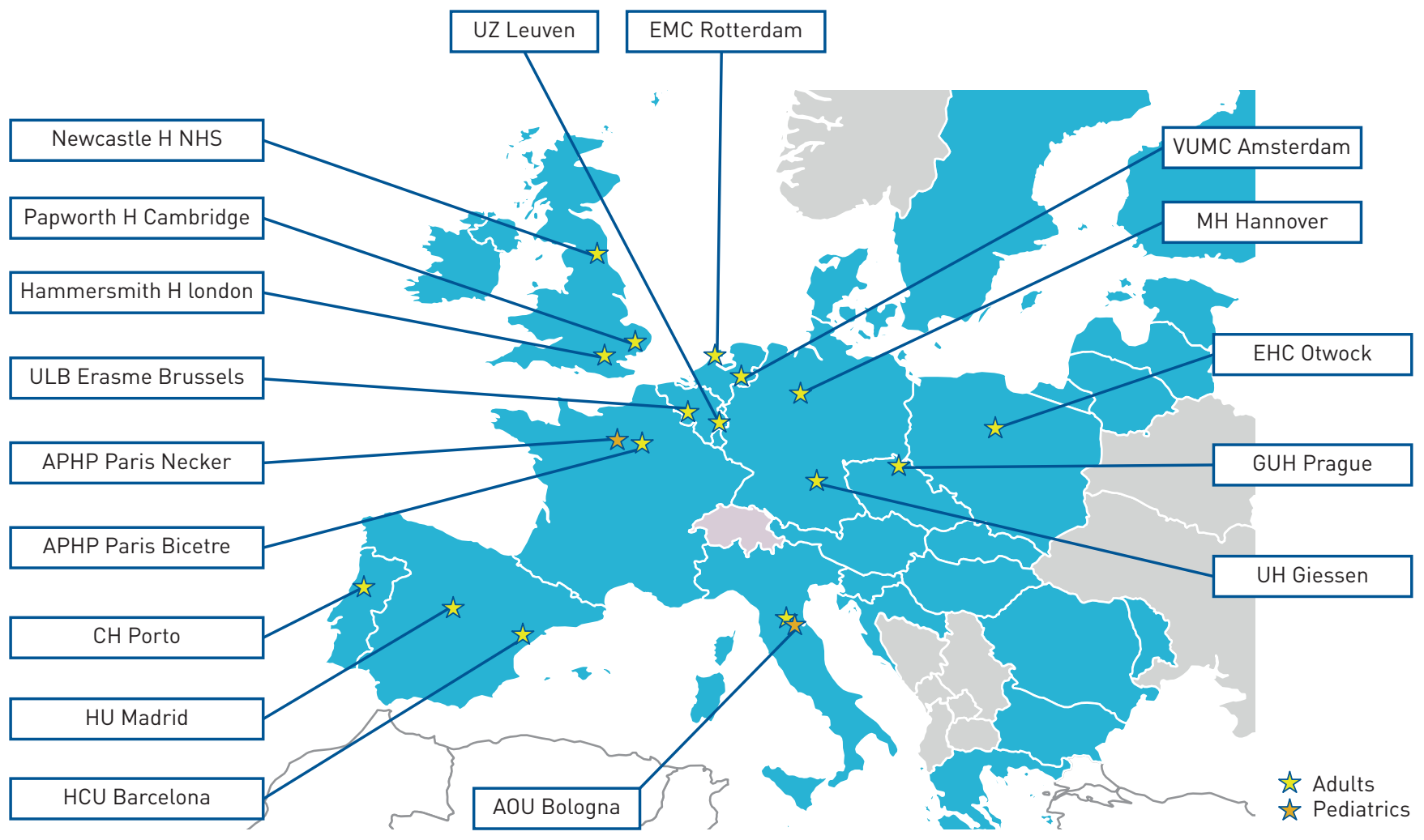

FIGURE 1 Healthcare providers constituting the pulmonary hypertension core network of ERN-Lung. 
FIGURE 2 ERN-Lung pulmonary hypertension core network registry survey.

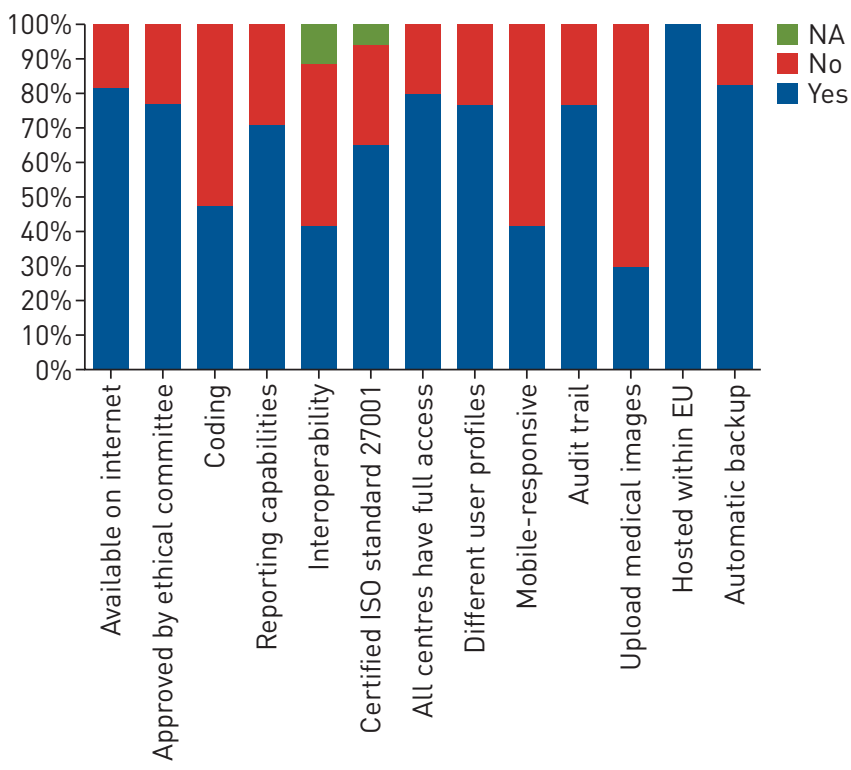

(82\%), they were either local $(41 \%)$ or multicentre $(59 \%)$, with important differences in coding, interoperability, certification, the possibility to upload medical images, etc. That is why the first specific project, which is also an ERN-Lung objective, consists of implementing common data collection platforms integrating existing systems, database fusion with the building of a "big data" bank of European $\mathrm{PH}$ patients, data sharing with the ERN-Lung warehouse of Registries, and elaboration of electronic case report forms for further CRC projects. The architecture of this construction is described in figure 3.

For the second objective, we plan to collate an in-depth database of leading PH referral centres, some of them already identified as ERN-Lung-PH HCP, via a web-based survey focusing on 1) diagnostic, therapeutic and follow-up strategies; 2) patient volume; 3) team structure and expertise; and 4) existing cross-border activities. We shall collect data on access and use of high-level and innovative diagnostic tests in routine clinical care, such as metabolic exercise testing, exercise and fluid volume loading tests during cardiac catheterisation and advanced imaging techniques, such as magnetic resonance and strain imaging.

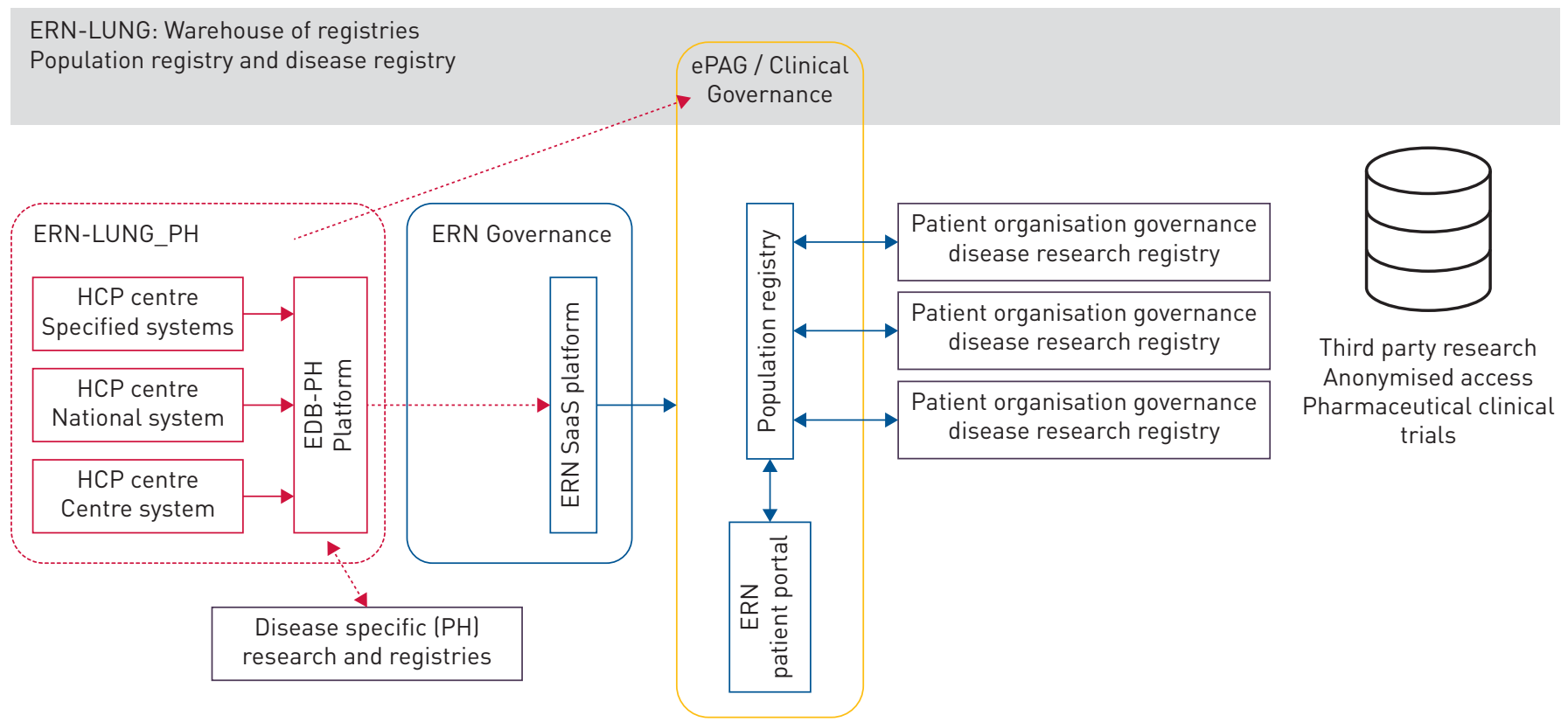

FIGURE 3 Architecture of the first work package of the Clinical Research Collaboration "PHAROS". ERN: European Reference Network; HCP: healthcare provider; SaaS: Scalable software as a Service for clinical management system to support ERN; ePAG: European Patient Advocacy Group (for each ERN disease group); EDB: European Database platform; PH: pulmonary hypertension. 
We shall also document the research activities and capabilities of institutions and networks, from routine biobanking of samples for 'omics to novel imaging techniques to support future large-scale, collaborative agnostic machine learning studies and early-phase and novel clinical trials.

The third project concerns the characterisation of $\mathrm{PH}$ patients with failing right ventricle (RV). Thereby, the PHAROS CRC aims to improve and structure the management of end-stage RV failure, to promote early recognition of RV adaptation profiles with new diagnostic tools, to improve our understanding on RV remodelling pathophysiology, and to identify new potential therapeutic targets for RV.

The fourth project concerns the phenotyping and treatment of patients with $\mathrm{PH}$ due to lung diseases (group 3 of the $\mathrm{PH}$ classification). In respiratory diseases the development of severe $\mathrm{PH}$ represents the end result of different trajectories. The identification and characterisation of these different trajectories will help us to understand the potential mechanisms involved in the pathogenesis of severe $\mathrm{PH}$ and identify potential ways to treat it in a particular patient. Further objectives are to evaluate the burden of severe $\mathrm{PH}$ associated with respiratory diseases and to quantify the number of patients with severe $\mathrm{PH}$ associated with respiratory diseases at the different sites, in order to design multicentre randomised controlled trials of $\mathrm{PH}$ treatment in respiratory diseases.

\section{Management structure of PHAROS}

The development of the CRC is led by a Steering Committee providing a balanced representation of scientific expertise and EU countries, also including patient representatives supported by the European Lung Foundation and early career members. The 17 ERN-Lung PH core members, plus affiliated and supporting members, represent the initial participants of PHAROS, building on the ERN prerequisite of expertise and patient data collection. The purpose of this editorial is to inform and invite $\mathrm{PH}$ expert centres who are interested by PHAROS to contact the CRC chairs. They could be able to join once structure and tools are well established.

Additional information on PHAROS can be obtained via the ERS Clinical Research Collaboration web page.

\section{Challenges}

Many challenges are still to be faced by PHAROS. Legal, regulatory and ethical aspects need to be considered on a case-by-case basis for each country/registry. As for all collaborative initiatives, success is conditioned by the commitment of the participants and the funding support from public or private sources. Conflict of interest may also appear as a consequence of clashes between national and pan-European interests.

Conflict of interest: O. Sitbon reports grants, personal fees and non-financial support from Actelion Pharmaceuticals, Bayer and MSD, grants from GlaxoSmithKline, personal fees from Gossamer Bio, Acceleron Pharmaceuticals and Ferrer, outside the submitted work. A. Reis reports grants, personal fees and non-financial support from Actelion Pharmaceuticals, outside the submitted work. M. Humbert reports grants and personal fees from Actelion, Bayer, GSK and Acceleron, personal fees from Merck and United Therapeutics, outside the submitted work. A. Vonk Noordegraaf is supported by the Netherlands CardioVascular Research Initiative (CVON-2012-08 PHAEDRA, CVON-2017-10 DOLPHIN-GENESIS) and the Netherlands Organization for Scientific Research (NWO-VICI: 918.16.610); and has received fees for lectures from Actelion, Johnson \& Johnson, Ferrer and Bayer in the past 3 years, and served as a member of the scientific advisory board of Morphogen-XI. M. Delcroix reports grants and personal fees for research, consultancy and lectures from Actelion/J\&J, personal fees for research, consultancy and lectures from Bayer, personal fees consultancy and lectures from MSD, personal fees for research from Reata, personal fees for research and consultancy from Bellarophon, personal fees for consultancy from Acceleron, outside the submitted work.

\section{References}

1 Simonneau G, Montani D, Celermajer DS, et al. Haemodynamic definitions and updated clinical classification of pulmonary hypertension. Eur Respir J 2019; 53: 1801913.

2 Galiè N, McLaughlin VV, Rubin LJ, et al. An overview of the 6th World Symposium on Pulmonary Hypertension. Eur Respir J 2019; 53: 1802148.

3 Galiè N, Humbert M, Vachiery J-L, et al. 2015 ESC/ERS Guidelines for the diagnosis and treatment of pulmonary hypertension. Eur Respir J 2015; 46: 903-975.

4 Galiè N, Humbert M, Vachiery J-L, et al. 2015 ESC/ERS Guidelines for the diagnosis and treatment of pulmonary hypertension: The Joint Task Force for the Diagnosis and Treatment of Pulmonary Hypertension of the European Society of Cardiology (ESC) and the European Respiratory Society (ERS). Eur Heart J 2016; 37: 67-119.

5 Grünig E, Weissmann S, Ehlken N, et al. Stress Doppler echocardiography in relatives of patients with idiopathic and familial pulmonary arterial hypertension: results of a multicenter european analysis of pulmonary artery pressure response to exercise and hypoxia. Circulation 2009; 119: 1747-1757.

6 Kovacs G, Herve P, Olschewski H. The pulmonary haemodynamics during exercise - research network (PEX-NET) ERS Clinical Research Collaboration: investigating the prognostic relevance of exercise haemodynamics. Eur Respir J 2019; 53: 1900458. 
7 Humbert $\mathrm{M}$, Sitbon $\mathrm{O}$, Chaouat $\mathrm{A}$, et al. Pulmonary arterial hypertension in France: results from a national registry. Am J Respir Crit Care Med 2006; 173: 1023-1030.

8 Humbert M, Sitbon O, Yaïci A, et al. Survival in incident and prevalent cohorts of patients with pulmonary arterial hypertension. Eur Respir J 2010; 36: 549-555.

9 Escribano-Subias P, Blanco I, López-Meseguer M, et al. Survival in pulmonary hypertension in Spain: insights from the Spanish registry. Eur Respir J 2012; 40: 596-603.

10 Ling Y, Johnson MK, Kiely DG, et al. Changing demographics, epidemiology, and survival of incident pulmonary arterial hypertension: results from the pulmonary hypertension registry of the United Kingdom and Ireland. Am J Respir Crit Care Med 2012; 186: 790-796.

11 Olsson KM, Delcroix M, Ghofrani HA, et al. Anticoagulation and survival in pulmonary arterial hypertension: results from the Comparative, Prospective Registry of Newly Initiated Therapies for Pulmonary Hypertension (COMPERA). Circulation 2014; 129: 57-65.

12 Baptista R, Meireles J, Agapito A, et al. Pulmonary hypertension in Portugal: first data from a nationwide registry. BioMed Res Int 2013; 2013: 1-8.

13 Boucly A, Weatherald J, Savale L, et al. Risk assessment, prognosis and guideline implementation in pulmonary arterial hypertension. Eur Respir J 2017; 50: 1700889.

14 Hoeper MM, Kramer T, Pan Z, et al. Mortality in pulmonary arterial hypertension: prediction by the 2015 European pulmonary hypertension guidelines risk stratification model. Eur Respir J 2017; 50: 1700740.

15 Kylhammar D, Kjellström B, Hjalmarsson C, et al. A comprehensive risk stratification at early follow-up determines prognosis in pulmonary arterial hypertension. Eur Heart J 2018; 39: 4175-4181.

16 McCollister D, Shaffer S, Badesch DB, et al. Development of the Pulmonary Arterial Hypertension-Symptoms and Impact (PAH-SYMPACT ${ }^{*}$ ) questionnaire: a new patient-reported outcome instrument for PAH. Respir Res 2016; 17: 72.

17 Yorke J, Corris P, Gaine S, et al. emPHasis-10: development of a health-related quality of life measure in pulmonary hypertension. Eur Respir J 2014; 43: 1106-1113.

18 Brightling C, Genton C, Bill W, et al. ERS Clinical Research Collaborations: underpinning research excellence. Eur Respir J 2018; 52: 1801534.

19 Noordegraaf A V, Delcroix M, Jiminez D, et al. Assembly 13: placing the pulmonary circulation in the heart of ERS. Breathe 2019; 15: 88-89.

20 Humbert M, Wagner TO. Rare respiratory diseases are ready for primetime: from Rare Disease Day to the European Reference Networks. Eur Respir J 2017; 49: 1700085. 